\title{
Revealing metabolic mechanisms of interaction in the anaerobic digestion microbiome by flux balance analysis
}

Basile, Arianna; Campanaro, Stefano; Kovalovszki, Adam; Zampieri, Guido; Rossi, Alessandro; Angelidaki, Irini; Valle, Giorgio; Treu, Laura

Published in:

Metabolic Engineering

Link to article, DOI:

10.1016/j.ymben.2020.08.013

Publication date:

2020

Document Version

Peer reviewed version

Link back to DTU Orbit

Citation (APA):

Basile, A., Campanaro, S., Kovalovszki, A., Zampieri, G., Rossi, A., Angelidaki, I., Valle, G., \& Treu, L. (2020). Revealing metabolic mechanisms of interaction in the anaerobic digestion microbiome by flux balance analysis. Metabolic Engineering, 62, 138-149. https://doi.org/10.1016/j.ymben.2020.08.013

\section{General rights}

Copyright and moral rights for the publications made accessible in the public portal are retained by the authors and/or other copyright owners and it is a condition of accessing publications that users recognise and abide by the legal requirements associated with these rights.

- Users may download and print one copy of any publication from the public portal for the purpose of private study or research.

- You may not further distribute the material or use it for any profit-making activity or commercial gain

- You may freely distribute the URL identifying the publication in the public portal 
1 Revealing metabolic mechanisms of interaction in the anaerobic digestion

2 microbiome by flux balance analysis

3 Arianna Basile, Stefano Campanaro*, Adam Kovalovszki, Guido Zampieri,

4 Alessandro Rossi, Irini Angelidaki, Giorgio Valle§, Laura Treu§

5

6 * corresponding author: stefano.campanaro@unipd.it

7 § equal contribution

Abstract

Anaerobic digestion is a key biological process for renewable energy, yet the mechanistic knowledge on its hidden microbial dynamics is still limited. The present work charted the interaction network in the anaerobic digestion microbiome via the full characterisation of pairwise interactions and the associated metabolite exchanges. To this goal, a novel collection of 836 genome-scale metabolic models was built to represent the functional capabilities of bacteria and archaea species derived from genome-centric metagenomics. Dominant microbes were shown to prefer mutualistic, parasitic and commensalistic interactions over neutralism, amensalism and competition, and are more likely to behave as metabolite importers and profiteers of the coexistence. Additionally, external hydrogen injection positively influences microbiome dynamics by promoting commensalism over amensalism. Finally, exchanges of glucogenic amino acids were shown to overcome auxotrophies caused by an incomplete tricarboxylic acid cycle. Our novel strategy predicted the most favourable growth conditions for the microbes, overall suggesting strategies to increasing the biogas production efficiency. In principle, this approach could also be applied to microbial populations of biomedical importance, such as the gut microbiome, to allow a broad inspection of the microbial interplays. 


\section{Keywords}

30 Anaerobic digestion/flux balance analysis/genome-scale metabolic models/microbial

31 interactions/renewable energy

\section{Introduction}

Microorganisms play an important role in all fields of biological relevance, ranging from human health (Clemente et al., 2012) to biotechnology (Lebuhn et al., 2015). In particular, diverse microbiomes may have various responsibilities, from causing diseases to influencing applied processes (e.g. biogas production) (Zhu et al., 2020) or the synthesis of polymeric substances (Chow et al., 2008). Microbial networks, however, are still poorly understood due to difficulties in isolating most of the microbial species and to the heterogeneous nature of their interactions (Muller et al., 2018). While the inspection of a core microbiome might reveal which species are the key players for a specific process (Faith, 2015), the role of rare members still remains to be clarified (Jousset et al., 2017). Microbial cooperation is extremely important for environmental niche colonization and completing complex activities (Stolyar et al., 2007), which single species could not perform independently (Hay et al., 2004). This is the case in anaerobic digestion (AD), which is a biotechnological process that produces a potent renewable energy carrier called biogas (Yentekakis and Goula, 2017). During biogas production, when acetoclastic methanogenic archaea are inhibited, a pivotal role is played by hydrogenotrophic methanogenic archaea and syntrophic acetate oxidizing bacteria (Mosbæk et al., 2016) (SAOB). An example is the mutualism between the hydrogen-utilizing methanogen Methanoculleus bourgensis and the SAOB Syntrophaceticus schinkii, [Clostridium] ultunense, and Tepidanaerobacter acetatoxydans (Westerholm et al., 2019). SAOB oxidise acetate to formate or to $\mathrm{H}_{2}$ and carbon dioxide $\left(\mathrm{CO}_{2}\right)$. The bacteria rely on archaeal activity, because acetate oxidation rapidly becomes endergonic when $\mathrm{H}_{2}$ accumulates (Stams 
and Plugge, 2009). Indeed, subsequently $\mathrm{H}_{2}$-utilizing methanogens convert these substrates to methane $\left(\mathrm{CH}_{4}\right)$ (Treu et al., 2018).

Although direct microbial cultivation and phenotyping experiments are essential to investigate metabolite exchanges and species interplays, this is not always possible. In fact, several microbes responsible for important biotechnological processes cannot be isolated and investigated using classical microbiological approaches because their specific growth demands are unknown, or because they cannot grow without the simultaneous existence of synergistic partners (Shlomi et al., 2007). In order to access the vast fraction of uncultivable microbes, genome centric metagenomics has given new possibilities to the scientific community (Parks et al., 2017).The extraction of genomes from complex assemblies allows the inference of metabolic pathways present in Metagenome Assembled Genomes (MAGs) (Nayfach et al., 2019), using the Kyoto Encyclopedia of Genes and Genomes database (KEGG) (Kanehisa et al., 2016) and/or EcoCyc (Keseler et al., 2017). Unfortunately, the use of gene annotation alone cannot fully describe the role of each microbial species in the global metabolic network mainly because many of the functions and reactions are still unknown (Zacher et al., 2014). Thus, a novel approach is needed for deciphering functional roles, microbial interactions and the exchange of molecules. Flux balance analysis (FBA) of in silico metabolic networks has recently emerged as one of the most effective methods to unveil microbial interplays (Magnúsdóttir et al., 2017). In fact, such metabolic models account for known intracellular processes (Budinich et al., 2017), as well as for metabolite uptake and secretion. This approach offers a reliable representation of the cross feeding occurring between community members (Khandelwal et al., 2013), and is able to predict how species gather in large consortia (Machado et al., 2020). However, previous studies relied on microbial consortia derived by $16 \mathrm{~S}$-based metagenomes to recover either the closest genomes or models publicly available in database. This assumption may limit the accuracy of the analysis as the correspondence between 16S-derived information and public data may be imprecise. Furthermore, microbial species can activate different pathways according 
to the growth medium and the relationship among different members of the same consortia. Metatranscriptomic results can be integrated to constrain the metabolic models and confirm previously flux balance analysis results. The ability to predict the structure and functioning of complex microbiomes is crucial for the study of organic matter degradation (Boon et al., 2014). The AD community is able to produce methane through a biologically mediated process, which is one of the oldest bioactivities on Earth (Sorokin et al., 2017). Anaerobic organic matter degradation naturally occurs in many ecological niches including, for example, the digestive tract of animals and anaerobic sediments (Liebetrau et al., 2019). Moreover, the biotechnological relevance is clear, as it can be exploited for biogas production. From an anthropocentric point of view, the AD microbiome organization can be visualized as a funnel (Campanaro et al., 2020) with methane as the final product. The funnel concept represents the progressive functional specialization, where the microbial players can be classified into four groups: hydrolytic, acidogenic and acetogenic bacteria, and methanogenic archaea (Campanaro et al., 2016). In the last few years, biotechnological applications in renewable energy and $\mathrm{CO}_{2}$ sequestration fields have been under active development due to the increasing environmental awareness, which is leading to a lower fossil fuel dependency and to a more intelligent management of natural resources (Hall and Scrase, 1998). During biogas production, the reactor efficiency can be increased through an accurate monitoring of process parameters, including temperature, mixing, reactor characteristics, feedstock composition (Angelidaki et al., 2018). All these factors can have a direct effect on the microbial community (Zhu et al., 2019). However, despite the microbiome's pivotal role in organic matter conversion into methane, there is still a lack of knowledge regarding the microbial influence on the process (Koch et al., 2019).

In order to unravel the microbial network and to clarify how process parameters can affect the microbiome structure, a FBA-based approach is proposed to explore metabolic exchanges in the AD community. Specifically, the objectives of the study were: a) to apply and develop a flux balance-based method for analysing coexistence 
114 dynamics in microbial consortia; b) to set-up a pipeline for the inspection of both 115 microbial pairwise interactions and metabolites exchanges considering also the

116 implementation of metatranscriptomic data to constrain the solution space; c) to 117 investigate the interdependencies among multiple species, d) to define a 118 comprehensive metabolic model of the AD microbiome. Additionally, bioinformatics 119 approaches were used to understand how cross-feeding varies according to the 120 different experimental conditions and simultaneous metabolite exchange among multiple species were investigated by analysing the flux network (Chaffron et al., 122 2010).

123

124

\section{Results}

The microbial species selected to be included as case study account for both archaea and bacteria and constitute the biggest known database of AD microbiome derived from metagenomic data (Campanaro et al., 2020). Of the reconstructed MAGs, only 836 have been considered for genome-scale metabolic model (GSMMs) reconstruction, which were identified as "high quality" regarding their genome completeness and contamination according to MIMAG guidelines (Bowers et al., 2017). These MAGs were taxonomically associated to 30 phyla involved in the AD process (Supplementary Table I) and represented the initial step of the investigation into interphylum and inter-kingdom interactions occurring in the anaerobic microbiome. The large-scale assessment of microbial relationships was performed in two stages, considering a feedstock rich in sugar and proteins in the former and the influence of external $\mathrm{H}_{2}$ injection in the latter (Treu et al., 2019). Subsequently, four different populations of the microbiome were used to analyse the occurring metabolic mechanisms and to determine the microbial interactivity. In this case, only the MAGs having a relative abundance higher than $0.001 \%$ were considered, based on the results of a previous experiment (Fontana et al., 2018) and using a feedstock rich in sugar and proteins. The first two populations were methanogenic, responsible for 
141 methane production before and after $\mathrm{H}_{2}$ injection, while the other two were acidogenic 142 and governed the production of acetate and other volatile fatty acids (VFA).

143 Upon reconstruction, each model underwent a battery of quality control tests to verify 144 biological and formal soundness. All models have full correspondence between reactions and genes, have demand reactions with fluxes in backward direction and pass the single gene deletion tests. The "leak tests" reports a score higher than 0.99 in all GSMMs (both with and without demand reactions) (Supplementary Table II). Moreover, we benchmarked our models against those from a previous large-scale collection of human gut microbiome GSMMs based on the MEMOTE tool (Lieven et al., 2020). This comparison highlighted a few systematic differences between the GSMM collections, probably due to the use of different model reconstruction software, some of which were already observed by Lieven and colleagues (Lieven et al., 2020) (Appendix and Figshare 10.6084/m9.figshare.12582746.v2). In particular, MEMOTE results revealed that AGORA models have a more complete GPR annotation and a lower number of mass-unbalanced reactions. However, models generated with CarveMe have a lower number of blocked reactions and so-called "orphan metabolites".

In total, 4132 unique metabolic reactions, involving 1580 different compounds, were identified. Each GSMM included on average 1391 \pm 245 reactions, $1012 \pm 152$ metabolites and $571 \pm 159$ genes (Fig. $1 \mathrm{~A}$ and Supplementary Table II). 112 metabolites (7\%) (e.g. L-Tryptophan, L-Valine, L-Tyrosine, Glycine, L-Methionine) and many cofactors (e.g. FAD, $\mathrm{FADH}, \mathrm{NMN}, \mathrm{NADP}^{+}, \mathrm{NADPH}_{2}$ ) were predicted in all models and were identified as "microbiome metabolite core", while 36 compounds were specific to individual models and were defined as "microbiome metabolite cloud". The presence and completeness of pathways responsible for carbohydrate and lipid metabolism have been tested in the metabolic models by tracking the corresponding reactions. Regarding carbohydrate metabolism, Actinomycetales sp. GSMM1485, Clostridiaceae sp. GSMM0156, Clostridiales spp. GSMM0297, GSMM0760, 
syntrophicus GSMM0017 were shown to have the complete anabolic and catabolic 171 pathways. Regarding lipids metabolism, "beta-Oxidation, acyl-CoA synthesis" and 172 "Phosphatidylethanolamine (PE) biosynthesis, PA => PS => PE" are complete in 18 173 GSMMs obtained from species belonging to the phylum Proteobacteria 174 (Supplementary Table II).

175 When considering the taxonomic assignment of MAGs at phylum level, some phyla had an average smaller number of genes, metabolites and reactions (Fig. 1A), with the microbial species assigned to the Synergistetes phylum showing the lowest number of genes recorded. On the other hand, Proteobacteria had the highest number of genes, followed by Acidobacteria, Actinobacteria and Armatimonadetes. This result shows consistency with microbes belonging to Proteobacteria being considered "generalist", as they can simultaneously perform multiple steps in the AD process, such as fatty acid degradation, butanoate metabolism, propionate metabolism, sulphate reduction and denitrification. This is in agreement with a previously reported study (Kitano, 2007).

A complete description of the metabolic potential present in the microbiome and the identification of new biochemical and physiological traits (both expected and nonintuitive) were accomplished by evaluating the 4132 biochemical reactions represented in the models. Only 33 "core reactions" were ubiquitously identified in all the microbes, which could be classified in three main categories: "transporters of simple molecules", "kinases" and "transferases". Moreover, methane biosynthesis was found in all the 29 archaeal species belonging to the Euryarchaeota phylum. A verification performed on the models by taking into account a set of 14 reactions revealed that Methanosarcina spp. (e.g. GSMM0042, GSMM0744, GSMM0883) can perform all the three methanogenic processes (acetoclastic, hydrogenotrophic and methylotrophic)

as

expected

while Euryarchaeota

sp. GSMM0884, Thermoplasmatales Incertae Sedis sp. GSMM1074 and Euryarchaeota sp. GSMM0778 have a complete set of enzymes only for the methylotrophic methanogenesis (Supplementary Table II). Among the others, Methanothermobacter 
spp. (e.g. GSMM0047 and GSMM0396) and Methanoculleus spp. (GSMM0046, GSMM0045) have been confirmed as hydrogenotrophs. Methanothrix soehngenii GSMM0064 and Methanosaeta sp. GSMM0125 have the complete acetoclastic pathway consistently with previous findings.

Conversely, 101 species-specific processes were defined as "shell reactions". For instance, the model of Clostridia sp. GSMM1577 was the only one predicting the use of coproporphyrinogen as an intermediate for heme biosynthesis (Dailey et al., 2015). Similarly, only Candidatus Riflebacteria sp. GSMM0304 could import and metabolize L-gulonate and D-tagatose (Raspor and Goranovič, 2008). These monosaccharides are epimers and precursors of glucose-6-phosphate and precursors respectively of dihydroxyacetone phosphate and pyruvate (Heinken \& Thiele, 2015). The unique characteristics of Candidatus Riflebacteria sp. GSMM0304 were related to CO utilization as a carbon source. In fact, this MAG was only present in a single experiment (Jing et al., 2017), suggesting its extreme specialization and possibly reflecting a low adaptability to survive in commonly utilised AD feedstocks. VFA, in particular acetate, propionate and butyrate are crucial intermediate compounds for the production of methane (Molina et al., 2009), as well as indicators of process stability (Jacobi et al., 2009) and agents for process diagnosis (Ahring et al., 1995). It is therefore extremely important to have a balance in the concentration of VFA, avoiding excessive accumulation of these intermediates in anaerobic reactors. The fundamental role of VFA in the AD system means that the possibility to estimate the intermediate compounds production and exchange in the AD microbiome is of great interest to tackle determinants of process instability (Liao et al., 2016). For this reason, the presence of genes involved in VFA production and utilization has been investigated in all the models (Fig. 1B). While some VFA, such as hexanoic, isovaleric and valeric acid were absent in the models, acetate was noticeably abundant, evidencing its association with the core microbial metabolism. According to the gene presence, the bacterial Wood-Ljungdahl pathway was identified as complete in nearly 
228 in 821 GSMMs, while butyrate in 693 (mainly in models of Bacteroidetes and 229 Synergistetes species), which were respectively 98 and $82 \%$ of all GSMMs 230 considered. In particular, propionate was involved in the biosynthesis of different key 231 compounds belonging to the glycolytic pathway, including succinate, fumarate and 232 oxaloacetate (Stryer, 1995). Assuming that the importance of different VFA in the 233 cross-feeding process can be determined by the fraction of microbes which are able 234 to exchange them, butyrate was probably one of the most relevant, since it was 235 exchanged by 635 species. Propionate was exported by a lower number of microbes, 236 suggesting a lower potential in cross-feedings. Among all VFA, isobutyrate was the 237 rarest, being present in 61 models (or 7\% of all GSMM) only. These results suggest 238 that the cross-feeding of butyrate is common, consequently redundant in the 239 microbiome, while the role of the few species involved in propionate exchange is 240 crucial. Accordingly, an alteration in the abundance of the propionate producers might 241 trigger a cascade effect, resulting in the loss of species utilizing this VFA to produce 242 other important intermediate metabolic compounds, such as fumarate and 243 oxaloacetate. 

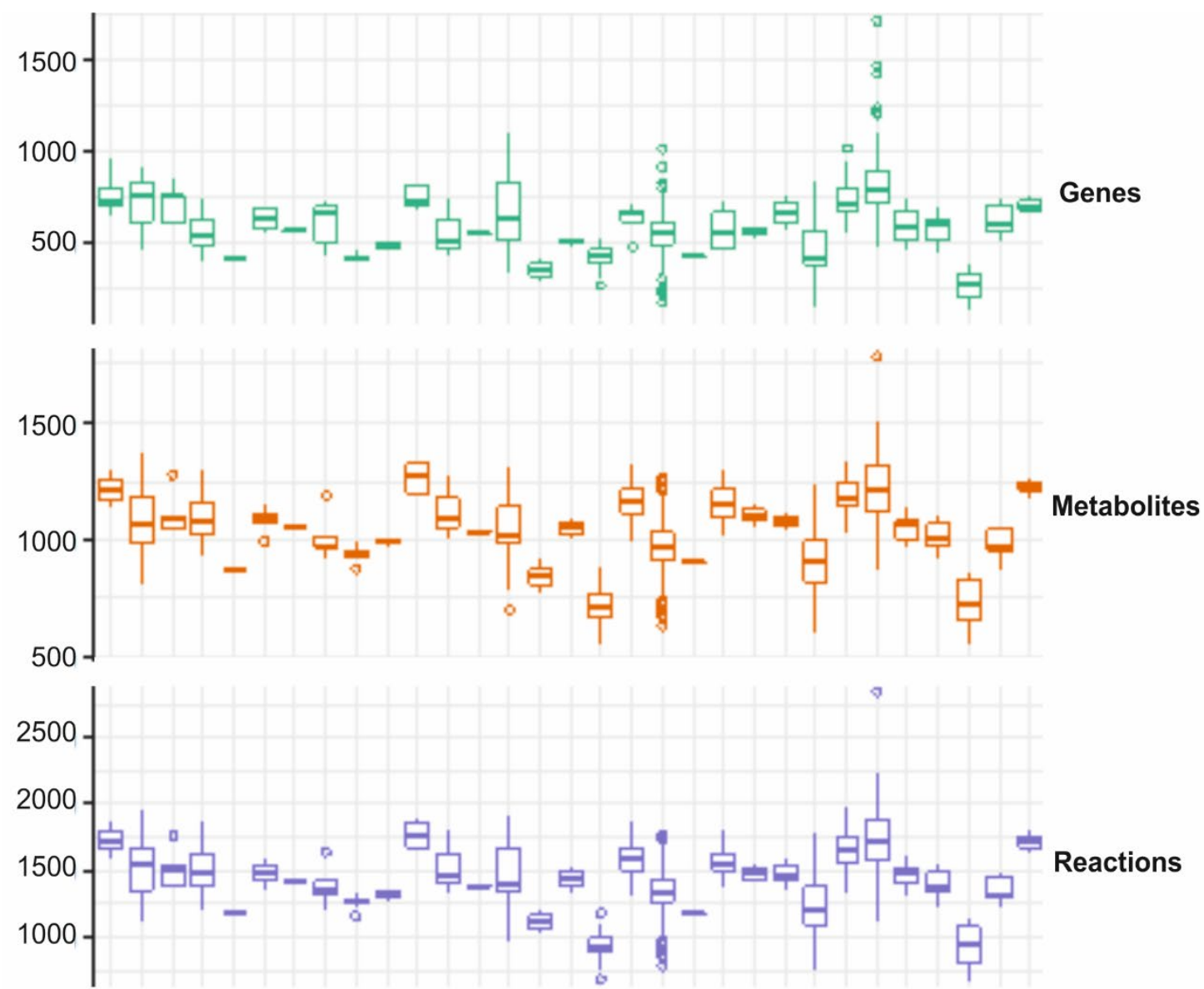

0000000000000000000000000000000 Acetate n-C2:0

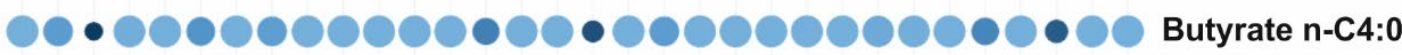
00000000000000000000000000000 Propionate n-C3:0
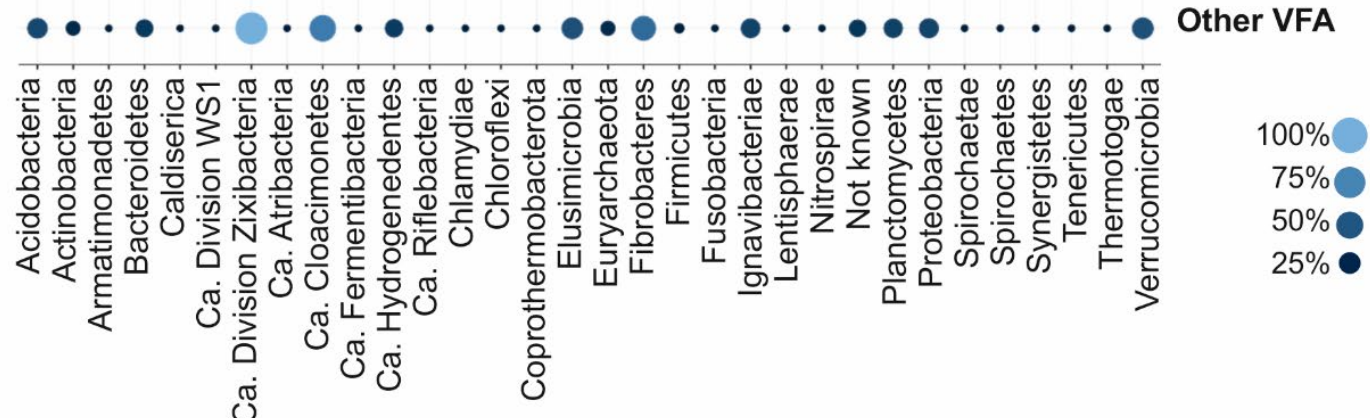

245 Fig. 1. General information of the metabolic models at phylum level.

246 Distribution of the number of genes, reactions and metabolites composing the reconstructed 247 genome-scale metabolic models and presence of specific volatile fatty acids, divided by 248 phylum. Whiskers show the minimum and maximum values. Values below "Q1 - $1.5 \times$ IQR" 249 and above "Q3 + 1.5 x IQR" are plotted as outliers (Q1: first quartile, Q3: third quartile, IQR: interquartile range). Colour and diameter of the circles depend on the fraction of microbes predicted to synthesize specific VFA. 


\subsection{Global landscape of pairwise interactions}

253 The potential of 349,030 microbial couples being involved in positive or negative 254 relationships was systematically investigated. In order to perform this investigation, 255 PAirwise INteractivity analysis (PA.IN.), a computational framework for the prediction 256 of interspecies behaviour was developed. Using the framework, different conditions 257 (including hydrogen concentration) were investigated, obtaining in total 698,060 pairs. 258 In the present work it was found impractical to perform simulations for interactions with 259 order higher than two on 836 models due to computational and time effort, despite this 260 type of analysis was previously applied (Machado et al., 2020).

261 Results revealed a variety of trade-offs within the microbe pairs depending on both the 262 metabolic potential and the imposed environmental conditions. A comparative 263 inspection of the relationships occurring between the 836 microbes, including those 264 obtained for species belonging to the "rare biosphere", was carried out to estimate the reciprocal potential impact of each species on the growth rate. This analysis was performed considering a diet designed "ad hoc" to simulate reactors' feedstock rich in sugar and proteins (the exact medium composition is reported in subsection 4.2). Not all the GSMM groups could grow individually on this specific feedstock; however, all the models showed non-zero growth in at least one of the possible couples. The feedstock components description was obtained integrating data from the $\mathrm{VMH}$ database (Noronha et al., 2019) with measurements conducted on the "real" feeding substrate (Fontana et al., 2018). Additionally, results on the microbial community composition were obtained for the same feedstock, but for two different growth conditions, one specifically characterized by external $\mathrm{H}_{2}$ injection. Hydrogen was supplemented to enhance the hydrogenotrophic methanogenesis and, consequently, to increase the methane content in the biogas, promoting the most important step of

277 the process (Nugent et al., 2013). Due to the relevant role of $\mathrm{H}_{2}$, both the shift in the 278 functional activity of single microbes after injection and its influence on the microbial 279 cross-talks were inspected. 
Based on the modifications in the growth rate of a species occurring while engaging with another member of the microbiome, six different patterns were determined: parasitism (+/-), commensalism (+/0), neutralism (0/0), amensalism (-/0), competition $(-/-)$ and mutualism (+/+) (where: + is positive, - is negative and 0 is no effect on each other between organisms) (Heinken and Thiele, 2015). When the coexistence negatively influences one of the two partners (growth rate decreases equal to or larger than $10 \%)$, the interplay is classified as negative, otherwise as positive. Neutralism is considered as a non-interaction, since the change in the growth rate derived from the microbial coexistence in the pair was included between $-10 \%$ and $+10 \%$.

In the community, commensalism was identified in $33 \%$ of the pairs, indicating a general positive reciprocal influence occurring between microbes (Fig. 2A). This behaviour, frequently detected in biodegradation processes (Campanaro et al, 2020), is mediated by cross-feeding which is indeed favouring synergies. Furthermore, among negative interactions, competition is the rarest, found only in $1.6 \%$ of the pairs (Appendix, Table S1). Similar results were obtained, considering the microbial species analyzed before using the cooperative tradeoff algorithm (Diener et al., 2020). This analysis confirmed that positive interactions are more frequent than negative ones (Supplementary Table III). Interestingly, the interactivity pattern identified is markedly different than that identified in other microbial communities where negative cross-talks are dominant. In fact, Heinken and coll. (Heinken and Thiele, 2015) found that parasitism is the most frequent meta-communication in human gut microbiome, while mutualism is the rarest. Notably, vessels in AD are strictly anaerobic while the gut is mainly microaerophilic, indicating that anoxicity promotes positive interspecies communication. An additional analysis was performed considering different thresholds for defining interactions: $\pm 1 \%, \pm 5 \%, \pm 15 \%$ and $\pm 20 \%$. The higher the threshold, the higher the number of neutralisms identified (Supplementary Table III). However, it can be concluded that the threshold was not influencing the detection of the specific behaviour characterizing the community. Despite setting the thresholds at $15 \%$ and $20 \%$ resulted in a higher number of neutral interplays in comparison to the 
309 commensalisms, negative interactions remain always lower in number than the 310 positive ones (even without considering neutralism as positive). Relevant trends 311 underlying the general organization of the community were identified at phylum level.

312 All phyla were involved in the six types of co-occurrences (except Caldiserica and 313 Chlamydia which include very few species) (Supplementary table III); however, some 314 preferential kinds of interaction were identified via a statistical analysis ( $p$-value<e-16, 315 chi-square test). In particular, some taxa are characterized by a specific pattern of 316 interactivity (validated by Pearson's residuals): Bacteroidetes, for example, are 317 frequently involved in neutralism and rarely in parasitism and amensalism 318 (Supplementary table IV). The opposite happens for Proteobacteria, whose species 319 are more involved in parasitic associations than in neutralistic. Spirochaetes, 320 Verrucomicrobia and Ignavibacteria among others, are the species interacting the 321 most through mutualistic association, while Caldiserica and Tenericutes are frequently 322 amensalistic (Supplementary table IV). 


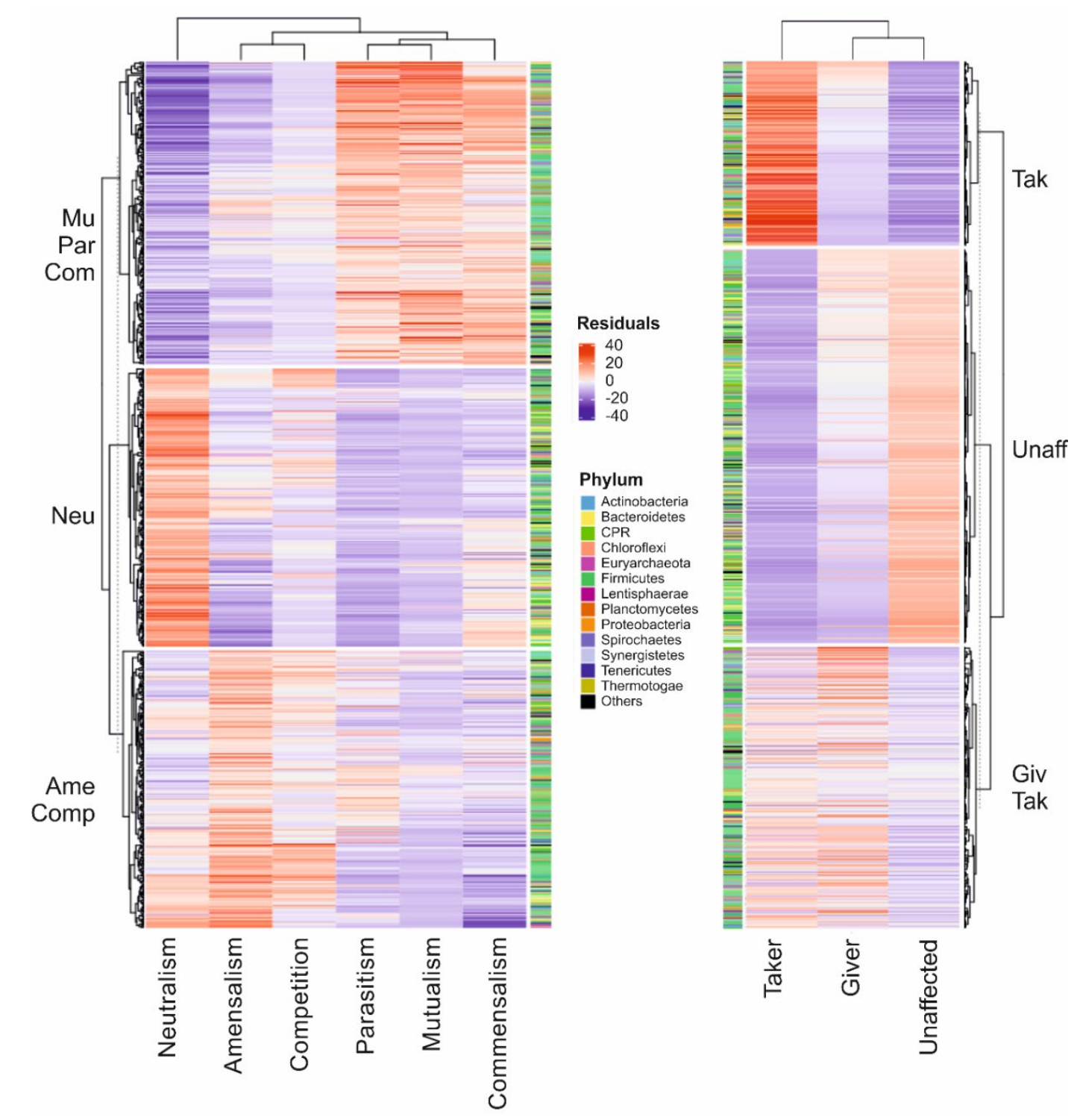

Fig. 2. Hierarchical clustering of the Pearson residual values.

325 Clustering has been performed both on interactivity and roles preferences profiles. The first 326 highlights three main groups of species having different interactivity profiles. "Neu" includes 327 species characterized mostly by neutralism and competition; "AmeComp" comprises those being predominantly amensalistic or competitive; "MuParCom" containing those that are 329 primarily mutualistic, parasitic and commensalistic. Clustering of the residual values performed on interactivity roles was performed in order to evidence species behaving as "givers" or "takers". Three main groups of species were identified: "Unaff" includes those 332 correlated neither with "giver", nor with "taker" behaviour (Neutral); "GivTak" consists of those with a heterogeneous behaviour in the interactions; "Tak" involving those behaving mainly as takers. 
General preferences at species level were defined by hierarchical clustering identifying three main behavioural clusters (Fig. 2): Neu, for microbes with preference for neutralism, AmeComp, for microbes with preference for amensalism and competition, MuParCom, for microbes with preference for mutualism, parasitism and commensalism. Euryarchaeota are mostly associated with Neu and MuParCom, with Methanosaeta and Methanomassiliicoccus species belonging to $\mathrm{Neu}$ and Methanothermobacter and Methanosarcina species belonging to MuParCom (Fig. 2).

342 This finding indicates that some archaeal species are more likely to express syntrophic

343 behaviour. Among others, Coprothermobacterota were also mainly found in 344 MuParCom, which could explain why Methanothermobacter wolfeii GSMM0047, Methanothermobacter sp. GSMM0492 and Methanosarcina thermophila GSMM0009 outcompete other archaea in the establishment of the community (Fontana et al., 2018). However, this behaviour was not limited to archaeal species, as the two dominant bacteria Coprothermobacter proteolyticus GSMM0002 and Defluviitoga tunisiensis GSMM0021 (up to $68 \%$ of combined relative abundance) adopted this behaviour too. Indeed, previous research performed on the metabolic pathways and the distribution of $D$. tunisiensis in the $A D$ environment suggested that it can be involved in syntrophic associations and metabolic exchanges with hydrogenotrophic methanogens (Maus et al., 2016). Nevertheless, D. tunisiensis is able to grow in pure culture, suggesting that syntrophies are not obligatory, albeit fruitful (Campanaro et al., 2018).

Another example for the importance of positive syntrophies was given by the PA.IN. inspection of Bifidobacterium crudilactis GSMM0001. This microbe presented a neutral behaviour in only $16 \%$ of the pairs, while on average other microbes remained unaffected in $51 \%$ of the pairs. This result could explain its great abundance, reaching $85 \%$ in the acidogenic community. A random sampling-based permutation test (see subsection 4.4 for additional details) revealed that highly abundant species like $B$. crudilactis GSMM0001 tend to show an overall positive behaviour, while low abundant species prefer amensalism. In general, most Bacteroidetes fell into the AmeComp 
cluster, and they were mainly unaffected by the interactions (growth rate variations were between $-10 \%$ and $10 \%$ ), indicating that these taxa use the same compounds as others, but without being outcompeted. The dominant biosphere is represented by a cooperative community conforming to the black queen hypothesis (Mas et al., 2016), while the rare microbes have overlapping nutritional requirements resulting in a more competitive community shaped as in the red queen hypothesis (Bonachela et al., 2017).

\subsection{Microbial classification based on individual interaction role}

372 Following the evaluation of microbial couples' behaviour during interactivity, it was possible to isolate and inspect the individual role of each microbe in the community by focusing on growth rate variations of the single species within each couple. In particular, the single microbes could benefit from the coexistence (takers) or were being negatively affected (givers). Species not influenced were defined as "unaffected". The significance of preferences for a specific role (givers or takers) was determined by chi-square statistics. Cluster analysis performed on residuals divided the species in three main clusters according to their behaviour (Fig. 2). Most abundant species were "takers" and included in the "Tak" cluster. This trend shows two relevant exceptions, i.e. the abundant species D. tunisiensis GSMM0021 and Epulopiscium sp. GSMM0167, both belonging to "GivTak". The rare biosphere was mainly included in cluster "Unaff", characterized by species with an unaffected behaviour (Neutral). Interspecies interactions could involve members of different phyla (interphyla) or could occur within the same phylum (intraphylum) (Fig. 3). Bacteria exclusively involved in interphyla relationships mainly belonged to Planctomycetes, Actinobacteria, Chloroflexi and Thermotogae, while other phyla showed either "intra" or "interphyla" interactivity. Notably, intraphylum mutualism was very rare, representing less than $0.001 \%$ of the total number of cross-feedings identified for a specific phylum (Fig. 3A). Possibly, this surprising feature is due to the similarities in the metabolic properties of species belonging to the same phylum, which tend to be involved in the same "trophic 
level" of the AD microbiome. This can result in species of the same phylum having a 393 higher level of competition and a lower number of mutualistic crosstalks, which in turn 394 were more frequent between microbes participating in different functional steps. On 395 the contrary, amensalism can occur intraphylum, revealing a competitive behaviour 396 between microbes of the same level contending for resources (Fig. 3B). The most prominent amensalistic tendency was observed between Firmicutes and Caldiserica, 398 whilst Firmicutes and Fusobacteria had the strongest mutualistic relationships.

A

399

400

401

402

403

404

405

406

407

408

409

410

411

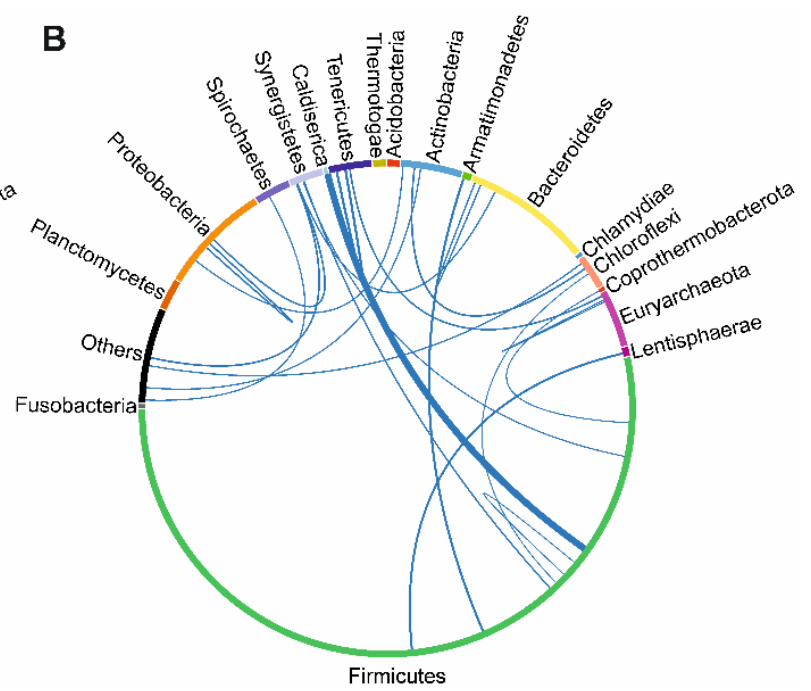

Fig. 3. Representation of interactions at phylum level.

(A) and (B) depict mutualistic and amensalistic interplays, respectively. Circumference arc length is proportional to the number of species in the corresponding phylum. Phyla represented by a low number of species were merged and named as "Others". Red (mutualistic) and blue (amensalistic) edge thickness represents the number of interplays normalized per phylum by number of species and having relative frequency higher than $0.001 \%$.

\subsection{Influence of externally supplied hydrogen on microbial interplay}

Hydrogen injection resulted in an increased number of mutualistic and commensalistic cross-talks as well as in fewer negative and neutral influences (Fig. 4, Supplementary

Table III), even though this effect was not widespread in the microbiome. In fact, despite the central role of hydrogen in many metabolic processes (it was present in 
412823 models and predicted to be exported by 509 species), its exogenous addition 413 impacted only $4.33 \%$ of the interacting microbial couples. The cooperative tradeoff 414 algorithm confirmed that hydrogen injection affects less than $5 \%$ of the couples and 415 drives the community to a more cooperative behaviour (Supplementary Table III). 416 Analyses performed in a methanogenic reactor supplemented with the feedstock 417 simulated in the present study, revealed that relative abundances of Leuconostoc sp. 418 GSMM0134 and Pseudomonas lactis GSMM0003 were markedly increased after $\mathrm{H}_{2}$ 419 injection (Fontana et al., 2018). Interestingly, PA.IN. results revealed that both 420 microbes switched from negative to positive interactivity after $\mathrm{H}_{2}$ addition. For 421 instance, the growth of Leuconostoc sp. GSMM0134 before $\mathrm{H}_{2}$ injection was negatively affected by 15 different members of the methanogenic community (of 22 in 423 total), which were probably competing for substrates (e.g. lactose, serine) or taking 424 advantage of by-products derived from substrate metabolism (e.g. L-Alanine, L425 Arabinose, Diacetyl, Succinate) (Supplementary Table V). External hydrogen addition 426 shifted this metabolic equilibrium, reducing the existing negative interactions to six and 427 enhancing the average growth rate of Leuconostoc sp. GSMM0134 from 0.08 to 0.11 $4281 / \mathrm{h}$. On the contrary, P. lactis GSMM0003 initially had a more diverse range of 429 interaction types that changed significantly upon hydrogen addition. Its average growth 430 rate increased slightly from 28.24 to $28.661 / \mathrm{h}$ and eight of its parasitic relationships 431 became mutualistic or commensalistic, with other five commensalistic relationships 432 shifting to parasitic (Supplementary table III). Although externally supplied $\mathrm{H}_{2}$ 433 influenced few selected couples, this finding suggests that changes induced 434 remarkable improvements in the syntrophies between bacteria and, consequently, 435 resulted in an increased methane production. 


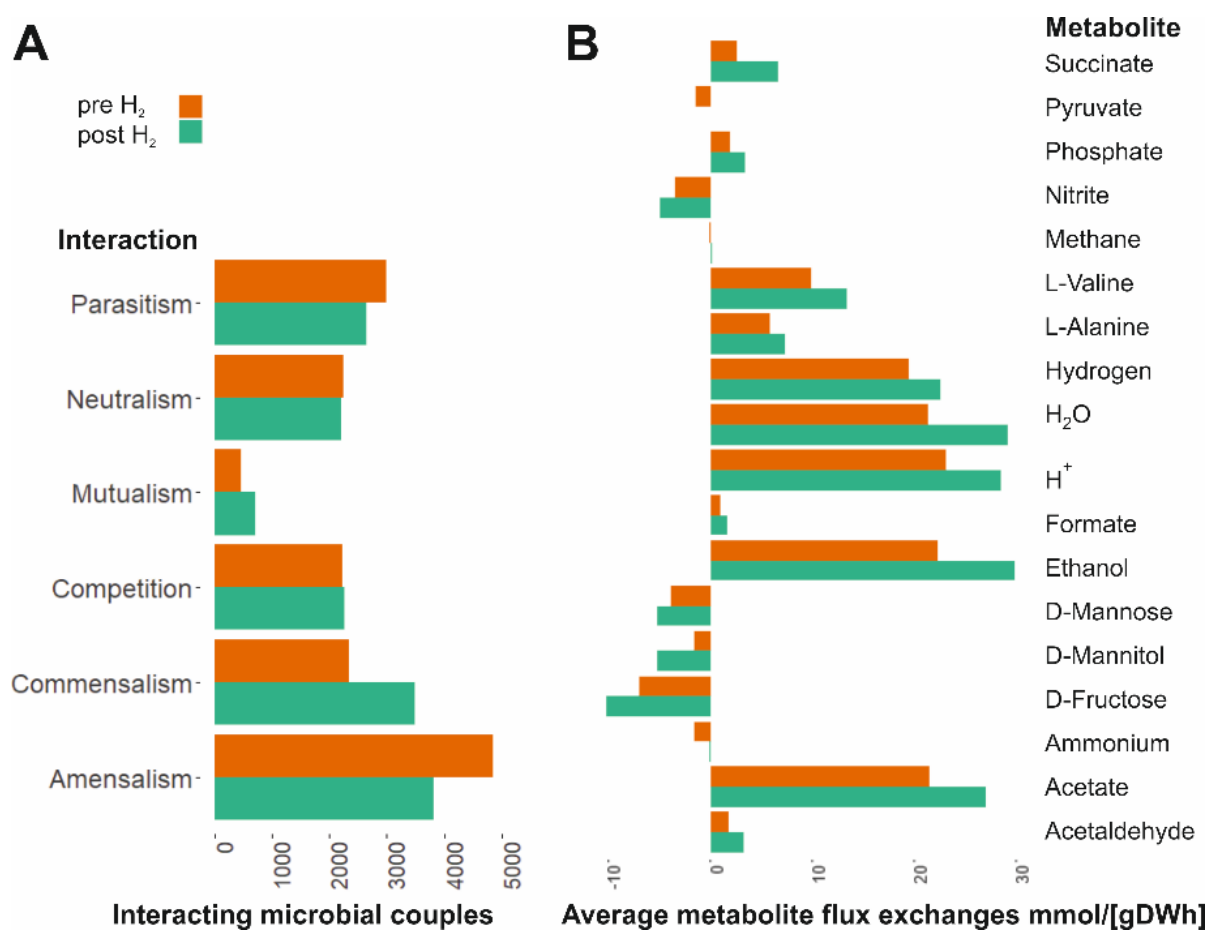

437 Fig. 4. Histograms representing the effect of $\mathrm{H}_{2}$ injection on the community.

438 (A), (B) effect on the microbiome interactivity profile and on average metabolite 439 production/consumption respectively. In panel A, shown interactions are only those that 440 change between the two conditions.

\section{$441 \quad 2.4$ From a global inspection to a detailed view of the interactions}

442 In order to substantiate and identify the interaction relations of the AD microcosms a 443 second case study was investigated. It represents the AD microbiome, including 444 metagenomic and metatranscriptomic data derived from the digestion of a simple 445 substrate composed by sugars and proteins in a two stage system (Fontana et al., 446 2018). Microbial communities inhabiting one methanogenic and one acidogenic 447 reactor were chosen in order to evaluate the compounds exchanged among species. 448 In particular, this experiment is taken as a case of study since it holds a combination 449 of features (i.e. small communities, high quality microbial genomes, simple feedstock) 450 that make it ideal for flux balance simulations. All microbes having relative abundance 451 greater than $0.01 \%$ in at least one of the two conditions were considered, representing 45269 and 10 species in methanogenic and acidogenic communities respectively. The 453 archaeal repertoire includes five species, one Methanosarcina (having the most 
454 versatile metabolism), two Methanothermobacter and two Methanoculleus (pure 455 hydrogenotrophs). The rest of the microbiome includes bacteria of Actinobacteria, 456 Bacteroidetes, Coprothermobacterota, Firmicutes, Proteobacteria, Synergistetes and 457 Thermotogae. Consistently with the experimental results, metabolite exchange 458 simulations indicated $10 \%$ increase in butyrate and methane, and $5 \%$ in acetate 459 production in the single-stage setup (methanogenic reactor). In the microbiome of 460 acidogenic reactor (two-stage system), the repertoire includes only bacteria of the 461 Firmicutes, Proteobacteria and Actinobacteria phyla and no archaea, due to the low $462 \mathrm{pH}$ (i.e. $3.9 \pm 0.1$ ) inhibiting the methanogenic process and hampering their growth. 463 Here, the simulations showed an increase in butyrate and acetate production and a 464 global increase of VFA, confirming previous findings about their importance. 465 Furthermore, the computational approach also estimated differences in the production 466 of several other compounds, which were not considered in the original study. In the 467 methanogenic reactor, for example, a decrease in the production of acetaldehyde, (S)468 propane-1,2-diol, pyruvate and ethanol was suggested by the simulations, following 469 hydrogen injection. Conversely, the uptake of L-aspartate, glycine, D-alanine and L470 glutamate was positively influenced subsequent to hydrogen injection (Supplementary 471 Table V). 


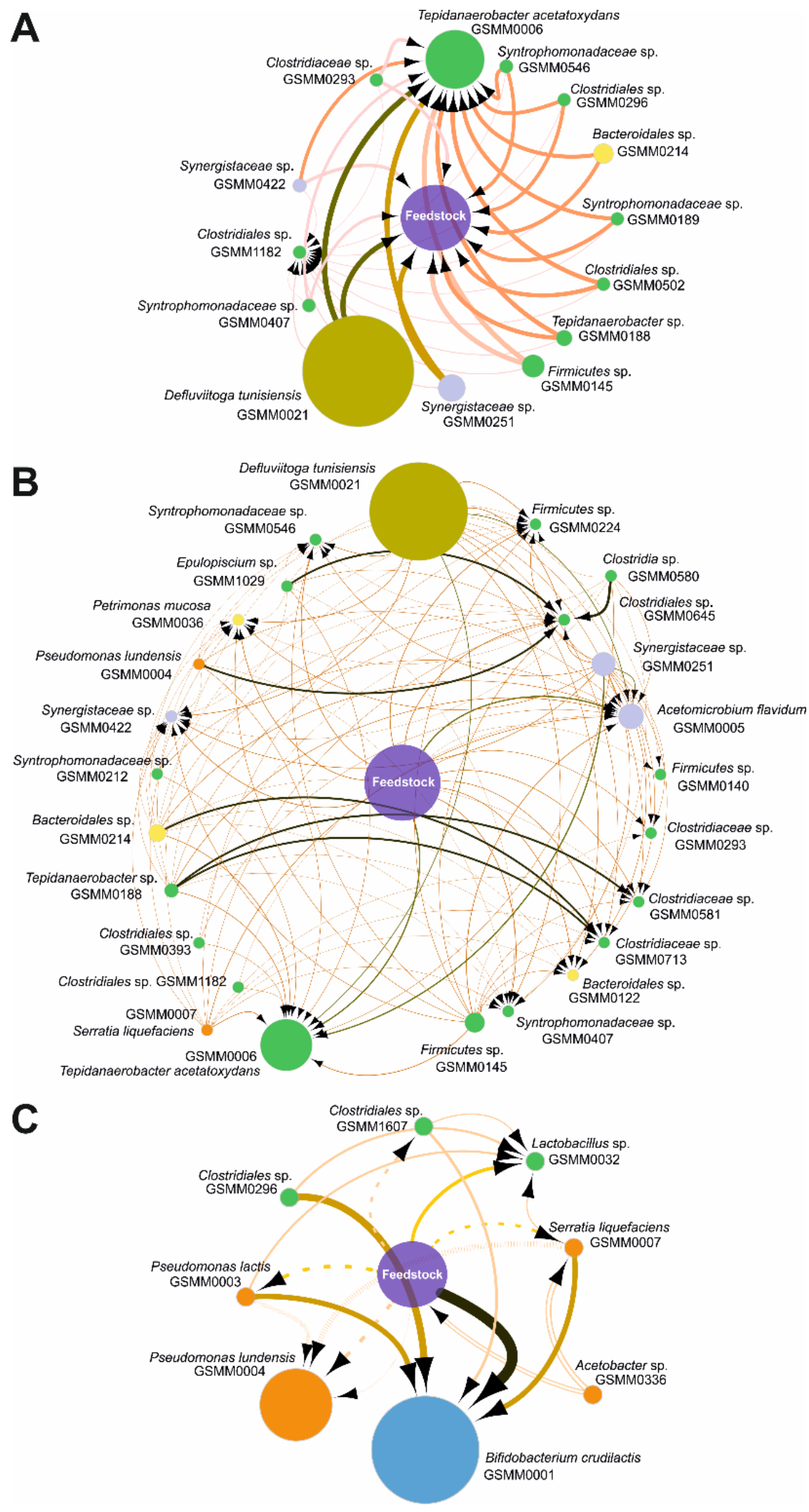

473 Fig. 5. Visualization of metabolic exchanges occurring between microbes present in the 474 reactor and / or with the medium. 
A-C. Circles represent the single microbes, the diameter is proportional to the species

476

477

478

479

480

481

482

483

484

485

486

487

488

489

490

491

492

493

494

495

496

497

498

499

500

501

502 abundance and the colour denotes the phylum. The medium is represented by the purple circle in the middle. Results for selected glucogenic amino acids such as glycine (A), and valine $(B)$ are reported. In panel $(C)$, key compounds related to the $A D$ process, such as acetate (dashed lines), acetaldehyde (solid lines), formate (parallel lines) and hydrogen (vertical slashed lines), are shown. Arrows thickness is proportional to the fluxes between species. Exchanges occurring in the methanogenic $(A-B)$ and in the acidogenic $(C)$ reactors, respectively, are reported.

In order to verify a putative causal relationship between metabolic compound exchange and results obtained from microbial coexistence, a comparative analysis was performed. In the methanogenic reactor, out of 196 compounds exported by at least one of the microbes, only 58 (29.6\%) could be imported by other species and were predicted as "cross feedings". Among those, 18 metabolites were related to amino acid metabolism, to which a similar prediction was evidenced in other complex microbial communities by Parks and colleagues (Parks et al., 2017). Amino acid interdependence is strictly linked with the lack of TCA cycle intermediates (Thommes et al., 2019). However, this imbalance, causing auxotrophies, can be compensated by compounds exchanges between members of the community. Metabolic exchange (ME) analysis (see subsection 4.3 for additional details) underlined the relevant role of glucogenic amino acids such as glycine, valine, and glutamate in the AD microbiome (Fig. 5). These compounds can be converted into intermediates of the TCA cycle, like glycine, the precursor of pyruvate, which is then converted into acetyl-CoA. Other compounds, such as valine and glutamate, are respectively precursors of succinylCoA and alpha-ketoglutarate.

According to the FBA carried out on the microbial community growing in the methanogenic reactor, Defluviitoga tunisiensis GSMM0021 was the most efficient producer of glycine and valine (Fig. 5A, Fig. 5B and Supplementary Table V), as well as the most abundant species. Tepidanaerobacter acetatoxydans GSMM0006 was 
predicted as the main up-taker of the two metabolites and the second most abundant microbe. Additionally, P. Iundensis GSMM0004 produced L-proline, which was imported by B. crudilactis GSMM0001 and converted into alpha-ketoglutarate. Another potential syntrophy was found between Lactobacillus paracasei subsp. paracasei GSMM0008 that produced L-Valine, and Leuconostoc sp. that imported and converted it to succinyl-CoA (Fig. 6). Both alpha-ketoglutarate and succinyl-CoA are TCA cycle intermediates and can be transformed into adenosine triphosphate (ATP), carbon dioxide and the reducing agent NADH (Moat et al., 2002). This finding hints the presence of a meta-communication, which is relevant also in the acidogenic reactor. Another interesting result obtained from ME revealed that a simultaneous exchange of acetaldehyde and acetate was occurring in the acidogenic reactor (Fig. 5C). Serratia liquefaciens GSMM0007, P. lactis GSMM0003 and Clostridiales sp. GSMM1607 were the major up-takers of acetaldehyde and the main producers of acetate, implying that the latter compound is generated by the activity of acetaldehyde dehydrogenase in specific bacteria. B. crudilactis GSMM0001 was the most abundant microbe overall, absorbing the vast majority of acetaldehyde and hydrogen, and concurrently stressing

519 the key role of these compounds for the proper functioning of the acidogenic reactor.

520 A putative syntrophic association between Coprothermobacter proteolyticus GSMM0002 and Methanothermobacter wolfeii GSMM0047 was previously proposed

522 (Fontana et al., 2018). Considering the relevance of this syntrophy in the methanogenic process, it was chosen for a detailed analysis, using the new methodology developed and supported by RNA-seq data integration. Previous studies mentioned a cooperative coexistence between proteolytic anaerobic bacteria and hydrogen-converting methanogens, evidencing that this syntrophy can lead to an increased cell growth and a better degradation efficiency (Stams, 1994). The present analysis showed that the two species could indeed reciprocally influence the growth rate of one another, with C. proteolyticus GSMM0002 benefiting from this relationship 530 in both conditions analysed and M. wolfeii GSMM0047 remaining almost unaffected.

531 Furthermore, ME showed that C. proteolyticus GSMM0002 produced $\mathrm{H}_{2}$ and iron 
$532\left(\mathrm{Fe}^{2+}\right)$, which were subsequently used by M. wolfeii GSMM0047, partially in agreement 533 with FBA integrating simulation of population steady-state metabolism

534 (Supplementary Table III). However, a comparative evaluation performed with ME and 535 starting from the results obtained with PA.IN. revealed a more complex situation. Both 536 C. proteolyticus GSMM0002 and M. wolfeii GSMM0047 benefited from the presence 537 of several other Clostridiales species in commensalistic interactions. M. wolfeii 538 GSMM0047 obtained $\mathrm{H}_{2}$ and formate from Clostridiales species, while C. proteolyticus 539 GSMM0002 absorbed tryptophan, L-proline and L-isoleucine, which are precursors of 540 alpha-ketoglutarate: a compound with a key role in the TCA cycle (Niehaus et al., 541 2019). Above result was supported by previous work asserting that SAOB interplays are not simply dualistic as previously thought but are based on multiple cross-feeding 543 effects (Westerholm et al., 2019). Finally, the original study reported an increase in methane production after $\mathrm{H}_{2}$ injection, and proposed an archaeal species potentially responsible for the biogas upgrading process (Fontana et al., 2018). In support of this proposal, ME estimated that Methanoculleus thermophilus GSMM0046 was the 547 microbe that profited the most from injecting $\mathrm{H}_{2}$ in the reactors, establishing it as the main microbe responsible for the increased methane yield and purity. These results were confirmed by simulations implementing gene expression values used to constrain the solution space (Supplementary Table V). For example, the flux for methane production shifts from 111.73 to $396.35 \mathrm{mmol} /[\mathrm{gDWh}]$, indicating an increase subsequent to $\mathrm{H} 2$ addition. However, based on the simulations integrating steadystate metabolism, M. thermophilus GSMM0046 showed strong profiteer behaviour, further emphasised after additional hydrogen injection (Supplementary Table III). In particular, parasitic relationships may be due to an enhanced uptake of several key metabolites and co-factors (Appendix, Table S2) by the archaeon. These compounds, thus, resulted less available in the medium for the other members of the community. Since there is not a universally accepted method to perform MFB community simulations and due to potential biases of the different approaches, some discrepancies are expected; however the key patterns obtained are in agreement and 


\section{2 the AD microbiome.}

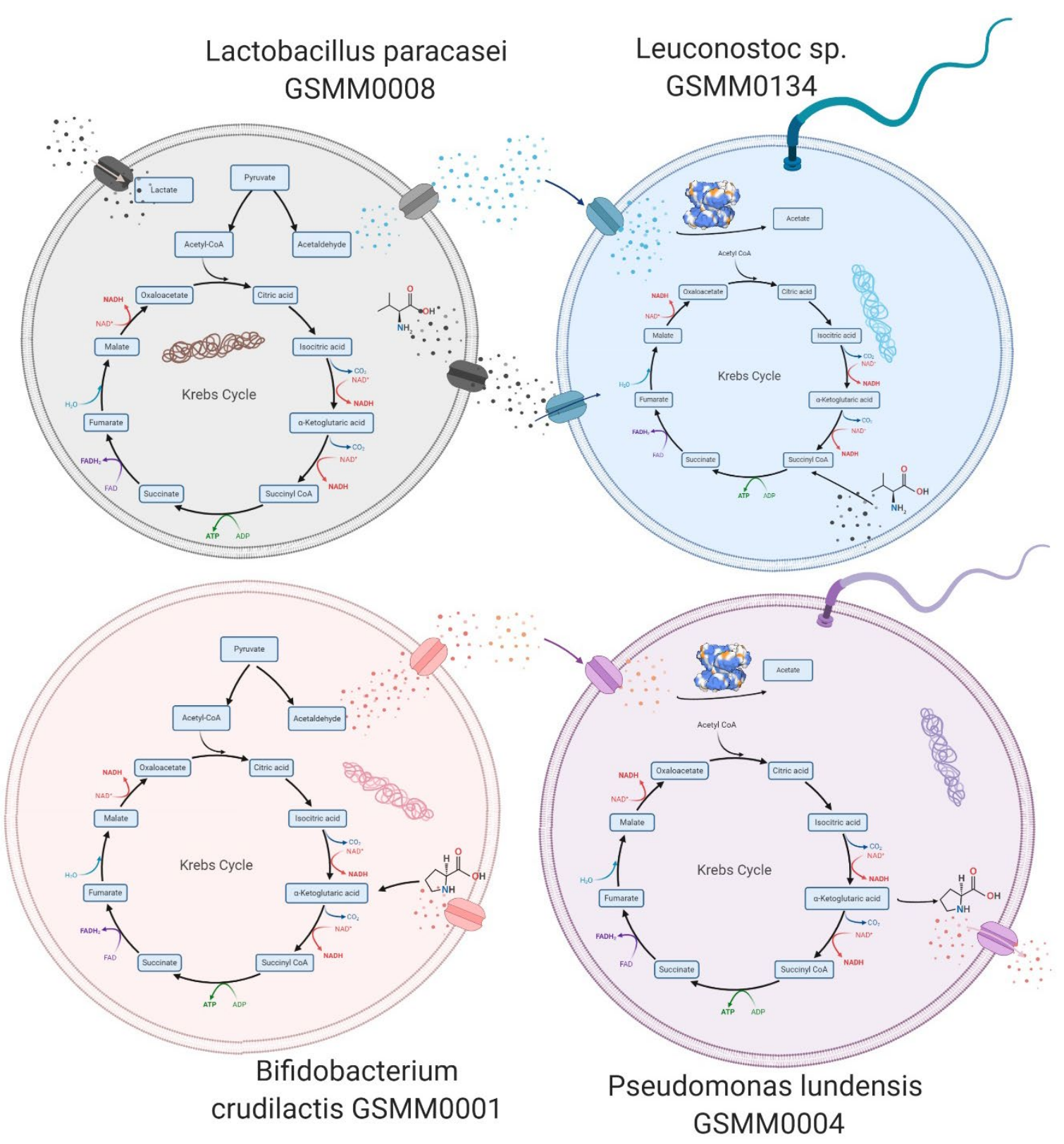

564 Fig. 6. Schematic representation of the TCA cycle in the four dominant species present in the 565 acidogenic reactor.

566 Results were integrated with the predicted amino acids exchanges influencing the TCA cycle.

567 The enzymatic structure represents the acetaldehyde dehydrogenase performing the 568 conversion of acetaldehyde into acetate. 


\section{Conclusions}

571 The major novelty of the current study is the combination of metabolic reconstruction

572 and modelling across hundreds of species for the detection of molecular mechanisms

573 behind their interactions at an unprecedented level of detail. To our knowledge, this is

574 the first attempt to massively generate models from high-quality MAGs, and to use flux

575 balance analysis for the inspection of a complex biologically mediated biogas

576 production system. The generation of metabolic models derived from known species,

577 and identified in the environment by means of $16 \mathrm{~S}$ rRNA similarity search, leaves

578 unresolved questions due to the presence of uncultivated microbes. On the contrary,

579 the approach proposed here paves the way to the use of FBA in the functional

580 investigation of the microbiome and allows a deeper understanding of all the species

581 present. The investigation of pairwise influence provided a comprehensive overview

582 of the microbial community involved in the anaerobic digestion process. From the

583 present investigation it emerges that reciprocal influence in the microbial growth rate

584 can be calculated in-silico and largely depends on competition/sharing for/of

585 metabolites. The used approach was successful in picking out strong differences in

586 interactivity roles between species that became prevailing in the community 587 evidencing how in this ecosystem two kinds of complementary communities coexist.

588 The consortium in the AD subsystem is stabilized by syntrophies and auxotrophies.

589 The accuracy of model creation and medium characterization enabled the 590 identification of key metabolites fluctuations between two experimental conditions 591 characterized by $\mathrm{H}_{2}$ injection. This resulted in differences in microbial growth 592 correlated with specific feedstock compositions. This analysis suggests that 593 investigation of pairwise interactivity can help in providing fundamental insights into 594 complex microbial populations (Weinrich et al., 2019). This approach is shedding light 595 on the network occurring in the $A D$ "black-box" and paves the way to a functional 596 prediction of the AD microbiome based on FBA. In perspective this newly developed 
597 procedure can target biogas production improvement through the design of "ad hoc" microbial consortia or by predicting substrate effects on the microbiome dynamics.

\section{4. Material and Methods}

\subsection{Description of the experiments}

601 For carrying out the modelling work and validating the metabolic models used in the present study, MAGs were obtained from the comparative analysis recently published

603 by Campanaro (Campanaro et al., 2020). The comparative analysis of more than 130

604 publicly available experiments from a range of Anaerobic Digestion engineered systems enabled the reconstruction of 5400 MAGs clustered into 1'600 different species with 837 of them identified as high quality according to MIMAG guidelines.

607 This repertoire is available in the database http://microbialgenomes.org/projects/biogasmicrobiome. From this dataset only 836 MAGs, identified as "high quality" regarding their genome completeness and contamination according to MIMAG guidelines (Bowers et al., 2017), have been considered for genome-scale metabolic model (GSMMs) reconstruction.

612 A comparison of single and two-stage AD setups was used from an earlier publication

613 (Fontana et al., 2018) included in the dataset mentioned above. The single stage reactor characterizes a complete $A D$ process fed with a mixed substrate of cheese whey and residues from cheese processing. On the other hand, the second system was composed of two reactors: the first reactor was fed as in the single-stage setup and used for the acidogenic step of $A D$, while its effluent was fed to the second reactor where methanogenesis took place. Both reactor systems had an operating temperature of $55 \pm 1{ }^{\circ} \mathrm{C}$ and were run without and with $\mathrm{H}_{2}$ injection (period I and II, respectively), in order to evaluate whether the injection of $\mathrm{H}_{2}$ modified the methane yield. For the present analysis, only the microbial communities from the methanogenic step of the single reactor and from the acidogenic step of the two-stage reactor setup were considered. The communities taken into account consisted of species having 
624 relative abundances higher than $0.01 \%$ in at least one of the four conditions analysed 625 (acidogenic of the two stage and methanogenic steps of the single stage setup, with 626 and without $\mathrm{H}_{2}$ injection), following (Fontana et al., 2018).

627 4.2 Genome-scale metabolic reconstruction and modelling of the anaerobic 628 digestion community

629 A tutorial was realized specifically for the replication of the analysis described in the present study (https://github.com/arianccbasile/ADinteractions).

631 Starting from quality-filtered MAGs, the genome scale metabolic models (GSMMs) for

632 the corresponding species were created with CarveMe v. 1.2. 1 (Machado et al., 2018) 633 using for each microbe the most appropriate universe (grampos, gramneg or archaea) 634 according to its taxonomy and the default gene matching parameters. The typical top635 down reconstruction approach implemented in CarveMe generated models with 636 standardized molecular weight of the biomass product. This is crucial, because without

637 proper normalization, hidden biases in biomass molecular weight can generate 638 significant simulation discrepancies (Siu H.J. Chan et al., 2017).

639 The solver used for GSMM reconstruction is Cplex (v. 12.8.0.0) (IBM, 2019). Sanity 640 checks were performed on all the metabolic models, testing twelve basic properties 641 through the COBRA toolbox (Brunk et al., 2018; Heirendt et al., 2019). Moreover, the consistency of all models was systematically assessed through the standardized

643 MEMOTE test suite (Lieven et al., 2020). Since AGORA (assembly of gut organisms 644 through reconstruction and analysis) models represent a golden standard in the field 645 of large-scale metabolic reconstructions, the analysis has been performed both on our 646 models and on AGORA models (Magnúsdóttir et al., 2017) to inspect the main 647 differences. To test the soundness of the models, the presence and completeness of 648 their pathways associated to carbohydrate and lipid metabolism have been verified by 649 considering the cross references in the microbial models and tracking the presence of specific reactions. Completeness of the KEGG modules was assessed for those

651 belonging to carbohydrate and lipid metabolism. For the inspection of methanogenesis 
and the Wood-Ljungdahl pathway (syntrophic acetate oxidation and/or acetogenesis), a manual validation of all the reactions was performed, taking into account relevant information derived from literature for the known species. Composition of the media has been obtained from the nutrition resource of the Virtual Metabolic Human database considering the following food entries: "Whey, acid, fluid", "Whey, acid, dried", "Whey, sweet, fluid", "Whey, sweet, dried". The corresponding exchange reactions have been refined through a manual work based on the experimental data previously reported (Fontana et al., 2018). The media composition is thoroughly described in the dedicated github. The medium composition was integrated in the model as nutrient constraints modifying accordingly the upper and lower bounds of export reactions through an in-house developed function ("apply_diet" in the github).

\subsection{Modelling of biological and metabolic interactions}

664 The analysis of the interactions among the different microbes was inferred using FBA.

665 For the current analysis Python and the COBRApy library (v. 0.17.1) (Ebrahim et al., 666 2013) were used. In order to use the metabolic models generated with CarveMe (v. 1.2.1), and to use the code with multiple processors the original MMinte (v. 1.0.3) (Mendes-Soares et al., 2016) code was adapted and reported in github as PA.IN. The generation of the communities follows the approach used in MMinte for the creation of multi-species stoichiometric models. This approach introduces a fictitious compartment that represents the extracellular environment shared by both species, and adds reactions allowing metabolites that are imported or secreted by each

673 individual species to be transformed into community metabolites. Singular growth 674 outcomes were computed by maximizing for total biomass production, setting the 675 maximal community growth as the objective function assuming that even for parasitic 676 or competitive microbes the best outcome is the maximal growth of the couple. The 677 growth rates for each species under defined nutrient conditions is estimated in 678 isolation and in the presence of another species, by running FBA in COBRApy. The 679 algorithm simultaneously maximizes for the biomass objective function of both 
680 GSMMs for the estimation of the growth rate of each species when coexisting with the other. The algorithm then knocks out all reactions of one species whilst maximizing

682 the biomass objective function of the other to simulate the growth rate of each species 683 individually. In this step the solver used is Glpk (Oki, 2012). A difference in the growth rate of $\pm 10 \%$ or more for at least a pair member, compared with the growth rate of the microbes grown separately, was considered significant to define the presence of an interaction. By comparing the growth rate of each microbe when coupled with another, 687 it was possible to distinguish six different types of interaction: parasitism, 688 commensalism, neutralism, amensalism, competition and mutualism. If at least one of 689 the two species was negatively affected by the interaction, the interplay was classified as negative, otherwise as positive. In some of these scenarios (namely Parasitism, Commensalism and Amensalism) each of the two species was classified either as a "giver" or a "taker", depending on the impact of the pairing on their growth. Interactions among the dominant members of the biosphere were verified using the cooperative tradeoff algorithm of Micom (v.0.10.1) (Diener et al., 2020), an approach mimicking the strategy implemented in OptCom (Zomorrodi and Maranas, 2012). By using this algorithm the individual growth is simultaneously at its maximum rate without diminishing the growth rate of other individuals (thus the term "cooperative"). Analysis of the metabolic exchanges among the different microbes has also been performed using MICOM integrating in the community model the microbial abundance as well and modifying the boundaries of the models according to the medium with an in-house developed script ("apply_diet" in the dedicated github). For each microbial pair, metabolite exchange rates were estimated through parsimonious FBA (Lewis et al., 2010) by setting the community biomass accumulation as objective. In this step, the solver used was Gurobi (v. 8) (Inc., 2014). Results were converted in tabular format and used to generate inputs file for Cytoscape software (3.2.1) (Demchak et al., 2014). Graphical representation of metabolic exchanges was performed with Cytoscape to simplify the interpretation of MICOM results. 
708

709

710

711

712

713

714

715

716

717

718

719

720

721

722

723

724

725

726

727

728

729

730

731

732

733

734

735

Metatranscriptomic data of twelve samples obtained from Fontana et al. (2018) were added to the simulation. Details of RNA extraction and standard raw RNA-seq data processing have been previously reported (Fontana et al., 2018). Gene expression analysis was performed using as reference the MAGs previously identified (Campanaro et al., 2020). Gene finding was performed using Prodigal (v2.6.2) (Hyatt et al., 2010) and reported in a previous study (Campanaro et al., 2020). RNA-seq reads were aligned on reference metagenome assembly using bowtie2 (v2.2.4) (Langmead and Salzberg, 2012) and the number of reads mapped per each gene was determined from the "bam" file using SeqMonk (v1.45.4) (Anders et al., 2015) using the options "opposing strand specific" and "apply transcript length correction". The metatranscriptomic data were implemented in the metabolic models as constraints readapting the code of METRADE (Angione and Lió, 2015) to the multispecies pairwise communities. Metabolic exchanges obtained starting from models with inner constraints derived from metatranscriptomic data were analysed as before. Metabolic models were deposited in the public repository figshare (10.6084/m9.figshare.12661496.v1).

Finally, we compared our results on metabolic exchanges obtained by Micom with those obtained through SteadyCom, a different approach for metabolic community modelling (Siu Hung Joshua Chan et al., 2017). In the SteadyCom simulations, the medium was integrated as microaerophilic, setting the aggregate oxygen uptake bound to $0.1 \mathrm{mmol} / \mathrm{h}$, upon finding that a completely anaerobic environment prevents most species from growing in isolation.

\subsection{Statistical analyses}

All the statistical analyses were carried out with R software v.3.5.2 (Breuer, 2017). The $\mathrm{X}^{2}$ test of Independence to characterise bacterial interactions across species was accomplished with chisq.test in the package stats. Pearson's residuals were obtained from the same function. Hierarchical clustering was applied via the ComplexHeatmap package (Gu et al., 2016), employing a squared Euclidean distance of the values 
between the 10th and the 90th percentile, thereby eliminating the influence of the

737 outliers (Appendix, Supplementary Methods, robust_dist). To verify the different

738 interactivity profile of abundant and rare community members, a permutation test was

739 carried out through the R base "sample" function, generating 2000 random

740 permutations (Appendix, Supplementary Methods, casual_campioning).

\section{Acknowledgements}

743 This work was financially supported by the "Budget Integrato della Ricerca 744 Dipartimentale" (BIRD198423) PRID 2019 of the Department of Biology of the 745 University of Padua, entitled "SyMMoBio: inspection of Syntrophies with Metabolic 746 Modeling to optimize Biogas Production". The PhD fellowship of AB is supported by 747 "Progetto di Eccellenza DiBio" of University of Padua. Authors want to thank Emiliano 748 Ciuffa for his significant contribution on linguistic revision of the manuscript and Daniel 749 Machado for the important suggestions provided during the revision process. A final 750 acknowledge to the Italian Consortium for Biotechnologies (CIB) for the support.

\section{Author contributions}

$753 \mathrm{AB}, \mathrm{LT}$ and SC conceived the experimental project; $\mathrm{AB}$ designed most of the 754 experimental strategy, developed code for the modelling analysis and performed the statistical analysis. All the authors wrote and revised the manuscript.

\section{Conflicts of interest}

758 The authors declare that they have no conflict of interest.

\section{References}


760

761

762

763

764

765

766

767

768

769

770

771

772

773

774

775

776

777

778

779

780

781

782

783

784

785

786

787

788

Ahring, B.K., Sandberg, M., Angelidaki, I., 1995. Volatile fatty acids as indicators of process imbalance in anaerobic digestors. Appl. Microbiol. Biotechnol. 43, 559565. https://doi.org/10.1007/BF00218466

Anders, S., Pyl, P.T., Huber, W., 2015. HTSeq-A Python framework to work with highthroughput sequencing data. Bioinformatics 31, 166-169. https://doi.org/10.1093/bioinformatics/btu638

Angelidaki, I., Treu, L., Tsapekos, P., Luo, G., Campanaro, S., Wenzel, H., Kougias, P.G., 2018. Biogas upgrading and utilization: Current status and perspectives. Biotechnol. Adv. https://doi.org/10.1016/j.biotechadv.2018.01.011

Angione, C., Lió, P., 2015. Predictive analytics of environmental adaptability in multiomic network models. Sci. Rep. https://doi.org/10.1038/srep15147

Bonachela, J.A., Wortel, M.T., Stenseth, N.C., 2017. Eco-evolutionary Red Queen dynamics regulate biodiversity in a metabolite-driven microbial system. Sci. Rep. 7. https://doi.org/10.1038/s41598-017-17774-4

Boon, E., Meehan, C.J., Whidden, C., Wong, D.H.J., Langille, M.G.I., Beiko, R.G., 2014. Interactions in the microbiome: Communities of organisms and communities of genes. FEMS Microbiol. Rev. https://doi.org/10.1111/15746976.12035

Bowers, R.M., Kyrpides, N.C., Stepanauskas, R., Harmon-Smith, M., Doud, D., Reddy, T.B.K., Schulz, F., Jarett, J., Rivers, A.R., Eloe-Fadrosh, E.A., Tringe, S.G., Ivanova, N.N., Copeland, A., Clum, A., Becraft, E.D., Malmstrom, R.R., Birren, B., Podar, M., Bork, P., Weinstock, G.M., Garrity, G.M., Dodsworth, J.A., Yooseph, S., Sutton, G., Glöckner, F.O., Gilbert, J.A., Nelson, W.C., Hallam, S.J., Jungbluth, S.P., Ettema, T.J.G., Tighe, S., Konstantinidis, K.T., Liu, W.T., Baker, B.J., Rattei, T., Eisen, J.A., Hedlund, B., McMahon, K.D., Fierer, N., Knight, R., Finn, R., Cochrane, G., Karsch-Mizrachi, I., Tyson, G.W., Rinke, C., Lapidus, A., Meyer, F., Yilmaz, P., Parks, D.H., Eren, A.M., Schriml, L., Banfield, J.F., Hugenholtz, P., Woyke, T., 2017. Minimum information about a single amplified genome (MISAG) and a metagenome-assembled genome (MIMAG) of bacteria 
Breuer, J., 2017. R (Software), in: The International Encyclopedia of Communication Research Methods. pp. 1-2. https://doi.org/10.1002/9781118901731.iecrm0201

Brunk, E., Sahoo, S., Zielinski, D.C., Altunkaya, A., Dräger, A., Mih, N., Gatto, F., 793 Nilsson, A., Preciat Gonzalez, G.A., Aurich, M.K., Prlic, A., Sastry, A., Danielsdottir, A.D., Heinken, A., Noronha, A., Rose, P.W., Burley, S.K., Fleming, R.M.T., Nielsen, J., Thiele, I., Palsson, B.O., 2018. Recon3D enables a threedimensional view of gene variation in human metabolism. Nat. Biotechnol. 36, 272-281. https://doi.org/10.1038/nbt.4072

798

799

800

801

802

803

804

805

806

807

808

809

810

811

812

813

814

815

816

817

Budinich, M., Bourdon, J., Larhlimi, A., Eveillard, D., 2017. A multi-objective constraintbased approach for modeling genome-scale microbial ecosystems. PLoS One 12. https://doi.org/10.1371/journal.pone.0171744

Campanaro, S., Treu, L., Kougias, P.G., De Francisci, D., Valle, G., Angelidaki, I., 2016. Metagenomic analysis and functional characterization of the biogas microbiome using high throughput shotgun sequencing and a novel binning strategy. Biotechnol. Biofuels 9. https://doi.org/10.1186/s13068-016-0441-1

Campanaro, S., Treu, L., Kougias, P.G., Luo, G., Angelidaki, I., 2018. Metagenomic binning reveals the functional roles of core abundant microorganisms in twelve full-scale biogas plants. Water Res. 140, 123-134. https://doi.org/10.1016/j.watres.2018.04.043

Campanaro, S., Treu, L., Rodriguez-R, L.M., Kovalovszki, A., Ziels, R.M., Maus, I., Zhu, X., Kougias, P.G., Basile, A., Luo, G., Schlüter, A., Konstantinidis, K.T., Angelidaki, I., 2020. New insights from the biogas microbiome by comprehensive genome-resolved metagenomics of nearly 1600 species originating from multiple anaerobic digesters. Biotechnol. Biofuels. https://doi.org/10.1186/s13068-02001679-y

Chaffron, S., Rehrauer, H., Pernthaler, J., Von Mering, C., 2010. A global network of coexisting microbes from environmental and whole-genome sequence data. Genome Res. 20, 947-959. https://doi.org/10.1101/gr.104521.109 
818 Chan, Siu H.J., Cai, J., Wang, L., Simons-Senftle, M.N., Maranas, C.D., 2017. 819 Standardizing biomass reactions and ensuring complete mass balance in 820 genome-scale metabolic models. Bioinformatics 33, 3603-3609. 821 https://doi.org/10.1093/bioinformatics/btx453

822 Chan, Siu Hung Joshua, Simons, M.N., Maranas, C.D., 2017. SteadyCom: Predicting 823 microbial abundances while ensuring community stability. PLoS Comput. Biol. 13. 824 https://doi.org/10.1371/journal.pcbi.1005539

Chow, D., Nunalee, M.L., Lim, D.W., Simnick, A.J., Chilkoti, A., 2008. Peptide-based 826 biopolymers in biomedicine and biotechnology. Mater. Sci. Eng. R Reports. https://doi.org/10.1016/j.mser.2008.04.004

Clemente, J.C., Ursell, L.K., Parfrey, L.W., Knight, R., 2012. The impact of the gut

Dailey, H.A., Gerdes, S., Dailey, T.A., Burch, J.S., Phillips, J.D., 2015. Noncanonical 832 microbiota on human health: An integrative view. Cell. https://doi.org/10.1016/j.cell.2012.01.035 coproporphyrin-dependent bacterial heme biosynthesis pathway that does not use protoporphyrin. Proc. Natl. Acad. Sci. U. S. A. 112, 2210-2215. https://doi.org/10.1073/pnas.1416285112

Demchak, B., Hull, T., Reich, M., Liefeld, T., Smoot, M., Ideker, T., Mesirov, J.P., 2014. Cytoscape: The network visualization tool for GenomeSpace workflows. F1000Research 3. https://doi.org/10.12688/f1000research.4492.1

Diener, C., Gibbons, S.M., Resendis-Antonio, O., 2020. MICOM: Metagenome-Scale Modeling To Infer Metabolic Interactions in the Gut Microbiota. mSystems 5. https://doi.org/10.1128/msystems.00606-19

Ebrahim, A., Lerman, J.A., Palsson, B.O., Hyduke, D.R., 2013. COBRApy: COnstraints-Based Reconstruction and Analysis for Python. BMC Syst. Biol. 7. https://doi.org/10.1186/1752-0509-7-74

Faith, J.J., 2015. Bridging the knowledge gap: from microbiome composition to function. Mol. Syst. Biol. 11, 793. https://doi.org/10.15252/msb.20156045

Fontana, A., Kougias, P.G., Treu, L., Kovalovszki, A., Valle, G., Cappa, F., Morelli, L., 
Angelidaki, I., Campanaro, S., 2018. Microbial activity response to hydrogen injection in thermophilic anaerobic digesters revealed by genome-centric metatranscriptomics. Microbiome 6. https://doi.org/10.1186/s40168-018-0583-4

Gu, Z., Eils, R., Schlesner, M., 2016. Complex heatmaps reveal patterns and correlations in multidimensional genomic data. Bioinformatics 32, 2847-2849. https://doi.org/10.1093/bioinformatics/btw313

Hall, D.O., Scrase, J.I., 1998. Will biomass be the environmentally friendly fuel of the future?, in: Biomass and Bioenergy. pp. 357-367. https://doi.org/10.1016/S09619534(98)00030-0

Hay, M.E., Parker, J.D., Burkepile, D.E., Caudill, C.C., Wilson, A.E., Hallinan, Z.P., Chequer, A.D., 2004. Mutualisms and aquatic community structure: The enemy of my enemy is my friend. Annu. Rev. Ecol. Evol. Syst. https://doi.org/10.1146/annurev.ecolsys.34.011802.132357

Heinken, A., Thiele, I., 2015. Anoxic conditions promote species-specific mutualism between gut microbes In Silico. Appl. Environ. Microbiol. 81, 4049-4061. https://doi.org/10.1128/AEM.00101-15

Heirendt, L., Arreckx, S., Pfau, T., Mendoza, S.N., Richelle, A., Heinken, A., Haraldsdóttir, H.S., Wachowiak, J., Keating, S.M., Vlasov, V., Magnusdóttir, S., Ng, C.Y., Preciat, G., Žagare, A., Chan, S.H.J., Aurich, M.K., Clancy, C.M., Modamio, J., Sauls, J.T., Noronha, A., Bordbar, A., Cousins, B., El Assal, D.C., Valcarcel, L. V., Apaolaza, I., Ghaderi, S., Ahookhosh, M., Ben Guebila, M., Kostromins, A., Sompairac, N., Le, H.M., Ma, D., Sun, Y., Wang, L., Yurkovich, J.T., Oliveira, M.A.P., Vuong, P.T., El Assal, L.P., Kuperstein, I., Zinovyev, A., Hinton, H.S., Bryant, W.A., Aragón Artacho, F.J., Planes, F.J., Stalidzans, E., Maass, A., Vempala, S., Hucka, M., Saunders, M.A., Maranas, C.D., Lewis, N.E., Sauter, T., Palsson, B., Thiele, I., Fleming, R.M.T., 2019. Creation and analysis of biochemical constraint-based models using the COBRA Toolbox v.3.0. Nat. Protoc. https://doi.org/10.1038/s41596-018-0098-2

Hyatt, D., Chen, G.L., LoCascio, P.F., Land, M.L., Larimer, F.W., Hauser, L.J., 2010. 
876

877

878

879

880

881

882

883

884

885

886

887

888

889

890

891

892

893

894

895

896

897

898

899

900

901

902

903

904

Prodigal: Prokaryotic gene recognition and translation initiation site identification. BMC Bioinformatics 11. https://doi.org/10.1186/1471-2105-11-119

IBM, 2019. CPLEX Optimizer [WWW Document]. Ibm. URL https://www.ibm.com/dkda/analytics/cplex-optimizer

Inc., G.O., 2014. Gurobi Optimizer reference manual. Www.Gurobi.Com 6, 572.

Jacobi, H.F., Moschner, C.R., Hartung, E., 2009. Use of near infrared spectroscopy in monitoring of volatile fatty acids in anaerobic digestion. Water Sci. Technol. 60, 339-346. https://doi.org/10.2166/wst.2009.345

Jing, Y., Campanaro, S., Kougias, P., Treu, L., Angelidaki, I., Zhang, S., Luo, G., 2017. Anaerobic granular sludge for simultaneous biomethanation of synthetic wastewater and $\mathrm{CO}$ with focus on the identification of CO-converting microorganisms.

Water

Res.

126,

19-28. https://doi.org/10.1016/j.watres.2017.09.018

Jousset, A., Bienhold, C., Chatzinotas, A., Gallien, L., Gobet, A., Kurm, V., Küsel, K., Rillig, M.C., Rivett, D.W., Salles, J.F., Van Der Heijden, M.G.A., Youssef, N.H., Zhang, X., Wei, Z., Hol, G.W.H., 2017. Where less may be more: How the rare biosphere pulls ecosystems strings. ISME J. https://doi.org/10.1038/ismej.2016.174

Kanehisa, M., Sato, Y., Kawashima, M., Furumichi, M., Tanabe, M., 2016. KEGG as a reference resource for gene and protein annotation. Nucleic Acids Res. 44, D457-D462. https://doi.org/10.1093/nar/gkv1070

Keseler, I.M., Mackie, A., Santos-Zavaleta, A., Billington, R., Bonavides-Martínez, C., Caspi, R., Fulcher, C., Gama-Castro, S., Kothari, A., Krummenacker, M., Latendresse, M., Muñiz-Rascado, L., Ong, Q., Paley, S., Peralta-Gil, M., Subhraveti, P., Velázquez-Ramírez, D.A., Weaver, D., Collado-Vides, J., Paulsen, I., Karp, P.D., 2017. The EcoCyc database: Reflecting new knowledge about Escherichia coli K-12. Nucleic Acids Res. 45, D543-D550. https://doi.org/10.1093/nar/gkw1003

Khandelwal, R.A., Olivier, B.G., Röling, W.F.M., Teusink, B., Bruggeman, F.J., 2013. 

PLoS One. https://doi.org/10.1371/journal.pone.0064567

907

908

909

910

911

912

913

914

915

916

917

918

919

920

921

922

923

924

925

926

927

928

929

930

931

932

933

Kitano, H., 2007. Towards a theory of biological robustness. Mol. Syst. Biol. https://doi.org/10.1038/msb4100179

Koch, S., Kohrs, F., Lahmann, P., Bissinger, T., Wendschuh, S., Benndorf, D., Reichl, U., Klamt, S., 2019. Redcom: A strategy for reduced metabolic modeling of complex microbial communities and its application for analyzing experimental datasets from anaerobic digestion. PLoS Comput. Biol. 15. https://doi.org/10.1371/journal.pcbi.1006759

Langmead, B., Salzberg, S.L., 2012. Fast gapped-read alignment with Bowtie 2. Nat. Methods 9, 357-359. https://doi.org/10.1038/nmeth.1923

Lebuhn, M., Weiß, S., Munk, B., Guebitz, G.M., 2015. Microbiology and molecular biology tools for biogas process analysis, diagnosis and control. Adv. Biochem. Eng. Biotechnol. 151. https://doi.org/10.1007/978-3-319-21993-6_1

Lewis, N.E., Hixson, K.K., Conrad, T.M., Lerman, J.A., Charusanti, P., Polpitiya, A.D., Adkins, J.N., Schramm, G., Purvine, S.O., Lopez-Ferrer, D., Weitz, K.K., Eils, R., König, R., Smith, R.D., Palsson, B., 2010. Omic data from evolved E. coli are consistent with computed optimal growth from genome-scale models. Mol. Syst. Biol. 6. https://doi.org/10.1038/msb.2010.47

Liao, J.C., Mi, L., Pontrelli, S., Luo, S., 2016. Fuelling the future: Microbial engineering for the production of sustainable biofuels. Nat. Rev. Microbiol. https://doi.org/10.1038/nrmicro.2016.32

Liebetrau, J., Sträuber, H., Kretzschmar, J., Denysenko, V., Nelles, M., 2019. Anaerobic digestion, in: Advances in Biochemical Engineering/Biotechnology. pp. 281-299. https://doi.org/10.1007/10_2016_67

Lieven, C., Beber, M.E., Olivier, B.G., Bergmann, F.T., Ataman, M., Babaei, P., Bartell, J.A., Blank, L.M., Chauhan, S., Correia, K., Diener, C., Dräger, A., Ebert, B.E., Edirisinghe, J.N., Faria, J.P., Feist, A.M., Fengos, G., Fleming, R.M.T., GarcíaJiménez, B., Hatzimanikatis, V., van Helvoirt, W., Henry, C.S., Hermjakob, H., 

J.J., König, M., Lakshmanan, M., Lee, D.Y., Lee, S.Y., Lee, S., Lewis, N.E., Liu, F., Ma, H., Machado, D., Mahadevan, R., Maia, P., Mardinoglu, A., Medlock, G.L., Monk, J.M., Nielsen, J., Nielsen, L.K., Nogales, J., Nookaew, I., Palsson, B.O., Papin, J.A., Patil, K.R., Poolman, M., Price, N.D., Resendis-Antonio, O., Richelle, A., Rocha, I., Sánchez, B.J., Schaap, P.J., Malik Sheriff, R.S., Shoaie, S., Sonnenschein, N., Teusink, B., Vilaça, P., Vik, J.O., Wodke, J.A.H., Xavier, J.C., Yuan, Q., Zakhartsev, M., Zhang, C., 2020. MEMOTE for standardized genomescale metabolic model testing. Nat. Biotechnol. 38, 272-276. https://doi.org/10.1038/s41587-020-0446-y

Machado, D., Andrejev, S., Tramontano, M., Patil, K.R., 2018. Fast automated reconstruction of genome-scale metabolic models for microbial species and communities.

Nucleic

Acids

Res.

46

7542-7553. https://doi.org/10.1093/nar/gky537

949

950

Machado, D., Maistrenko, O.M., Andrejev, S., Kim, Y., Bork, P., Patil, Kaustubh R., Patil, Kiran R., 2020. Polarization of microbial communities between competitive 951 952 2020.01.28.922583. https://doi.org/10.1101/2020.01.28.922583

Magnúsdóttir, S., Heinken, A., Kutt, L., Ravcheev, D.A., Bauer, E., Noronha, A., Greenhalgh, K., Jäger, C., Baginska, J., Wilmes, P., Fleming, R.M.T., Thiele, I., 2017. Generation of genome-scale metabolic reconstructions for 773 members of the human gut microbiota. Nat. Biotechnol. 35, 81-89. https://doi.org/10.1038/nbt.3703

Mas, A., Jamshidi, S., Lagadeuc, Y., Eveillard, D., Vandenkoornhuyse, P., 2016. Beyond the Black Queen Hypothesis. ISME J. https://doi.org/10.1038/ismej.2016.22 Sczyrba, A., König, H., Pühler, A., Schlüter, A., 2016. Genomic characterization of Defluviitoga tunisiensis L3, a key hydrolytic bacterium in a thermophilic biogas 
963

964

965

966

967

968

969

970

971

972

973

974

975

976

977

978

979

980

981

982

983

984

985

986

987

988

989

990

991

plant and its abundance as determined by metagenome fragment recruitment. J. Biotechnol. 232, 50-60. https://doi.org/10.1016/j.jbiotec.2016.05.001

Mendes-Soares, H., Mundy, M., Soares, L.M., Chia, N., 2016. MMinte: An application for predicting metabolic interactions among the microbial species in a community. BMC Bioinformatics 17. https://doi.org/10.1186/s12859-016-1230-3

Moat, A.G., Foster, J.W., Spector, M.P., 2002. Microbial Physiology, Microbial Physiology. https://doi.org/10.1002/0471223867

Molina, F., Ruiz-Filippi, G., Garcia, C., Lema, J.M., Roca, E., 2009. Pilot-scale validation of a new sensor for on-line analysis of volatile fatty acids and alkalinity in anaerobic wastewater treatment plants. Environ. Eng. Sci. 26, 641-649. https://doi.org/10.1089/ees.2007.0308

Mosbæk, F., Kjeldal, H., Mulat, D.G., Albertsen, M., Ward, A.J., Feilberg, A., Nielsen, J.L., 2016. Identification of syntrophic acetate-oxidizing bacteria in anaerobic digesters by combined protein-based stable isotope probing and metagenomics. ISME J. 10, 2405-2418. https://doi.org/10.1038/ismej.2016.39

Muller, E.E.L., Faust, K., Widder, S., Herold, M., Martínez Arbas, S., Wilmes, P., 2018. Using metabolic networks to resolve ecological properties of microbiomes. Curr. Opin. Syst. Biol. https://doi.org/10.1016/j.coisb.2017.12.004

Nayfach, S., Shi, Z.J., Seshadri, R., Pollard, K.S., Kyrpides, N.C., 2019. New insights from uncultivated genomes of the global human gut microbiome. Nature 568, 505-510. https://doi.org/10.1038/s41586-019-1058-x

Niehaus, L., Boland, I., Liu, M., Chen, K., Fu, D., Henckel, C., Chaung, K., Miranda, S.E., Dyckman, S., Crum, M., Dedrick, S., Shou, W., Momeni, B., 2019. Microbial coexistence through chemical-mediated interactions. Nat. Commun. 10. https://doi.org/10.1038/s41467-019-10062-x

Noronha, A., Modamio, J., Jarosz, Y., Guerard, E., Sompairac, N., Preciat, G., Daníelsdóttir, A.D., Krecke, M., Merten, D., Haraldsdóttir, H.S., Heinken, A., Heirendt, L., Magnúsdóttir, S., Ravcheev, D.A., Sahoo, S., Gawron, P., Friscioni, L., Garcia, B., Prendergast, M., Puente, A., Rodrigues, M., Roy, A., Rouquaya, 
992

993

994

995

996

997

998

999

1000

1001

1002

1003

1004

1005

1006

1007

1008

1009

1010

1011

1012

1013

1014

1015

1016

1017

1018

1019

1020

M., Wiltgen, L., Žagare, A., John, E., Krueger, M., Kuperstein, I., Zinovyev, A., Schneider, R., Fleming, R.M.T., Thiele, I., 2019. The Virtual Metabolic Human database: Integrating human and gut microbiome metabolism with nutrition and disease. Nucleic Acids Res. 47, D614-D624. https://doi.org/10.1093/nar/gky992

Nugent, P., Giannopoulou, E.G., Burd, S.D., Elemento, O., Giannopoulou, E.G., Forrest, K., Pham, T., Ma, S., Space, B., Wojtas, L., Eddaoudi, M., Zaworotko, M.J., 2013. Porous materials with optimal adsorption thermodynamics and kinetics for co2 separation. Nature 495, 80-84. https://doi.org/10.1038/nature11893

Oki, E., 2012. GLPK (GNU Linear Programming Kit), in: Linear Programming and Algorithms for Communication Networks. pp. 25-29. https://doi.org/10.1201/b12733-4

Parks, D.H., Rinke, C., Chuvochina, M., Chaumeil, P.A., Woodcroft, B.J., Evans, P.N., Hugenholtz, P., Tyson, G.W., 2017. Recovery of nearly 8,000 metagenomeassembled genomes substantially expands the tree of life. Nat. Microbiol. 2, 1533-1542. https://doi.org/10.1038/s41564-017-0012-7

Raspor, P., Goranovič, D., 2008. Biotechnological applications of acetic acid bacteria. Crit. Rev. Biotechnol. https://doi.org/10.1080/07388550802046749

Shlomi, T., Eisenberg, Y., Sharan, R., Ruppin, E., 2007. A genome-scale computational study of the interplay between transcriptional regulation and metabolism. Mol. Syst. Biol. 3. https://doi.org/10.1038/msb4100141

Sorokin, Di.Y., Makarova, K.S., Abbas, B., Ferrer, M., Golyshin, P.N., Galinski, E.A., Ciordia, S., Mena, M.C., Merkel, A.Y., Wolf, Y.I., Van Loosdrecht, M.C.M., Koonin, E. V., 2017. Discovery of extremely halophilic, methyl-reducing euryarchaea provides insights into the evolutionary origin of methanogenesis. Nat. Microbiol. 2. https://doi.org/10.1038/nmicrobiol.2017.81

Stams, A.J.M., 1994. Metabolic interactions between anaerobic bacteria in methanogenic environments. Antonie Van Leeuwenhoek 66, 271-294. https://doi.org/10.1007/BF00871644 
1021

1022

1023

1024

1025

1026

1027

1028

1029

1030

1031

1032

1033

1034

1035

1036

1037

1038

1039

1040

1041

1042

1043

1044

1045

1046

1047

1048

1049

Stams, A.J.M., Plugge, C.M., 2009. Electron transfer in syntrophic communities of anaerobic bacteria and archaea. Nat. Rev. Microbiol. https://doi.org/10.1038/nrmicro2166

Stolyar, S., Van Dien, S., Hillesland, K.L., Pinel, N., Lie, T.J., Leigh, J.A., Stahl, D.A., 2007. Metabolic modeling of a mutualistic microbial community. Mol. Syst. Biol. 3, 1-14. https://doi.org/10.1038/msb4100131

Stryer, L., 1995. Stryer Biochemistry, Biochemistry textbook.

Thommes, M., Wang, T., Zhao, Q., Paschalidis, I.C., Segrè, D., 2019. Designing Metabolic Division of Labor in Microbial Communities. mSystems 4. https://doi.org/10.1128/msystems.00263-18

Treu, L., Campanaro, S., Kougias, P.G., Sartori, C., Bassani, I., Angelidaki, I., 2018. Hydrogen-fueled microbial pathways in biogas upgrading systems revealed by genome-centric metagenomics.

Front.

Microbiol.

9. https://doi.org/10.3389/fmicb.2018.01079

Treu, L., Tsapekos, P., Peprah, M., Campanaro, S., Giacomini, A., Corich, V., Kougias, P.G., Angelidaki, I., 2019. Microbial profiling during anaerobic digestion of cheese whey in reactors operated at different conditions. Bioresour. Technol. 275, 375-385. https://doi.org/10.1016/j.biortech.2018.12.084

Weinrich, S., Koch, S., Bonk, F., Popp, D., Benndorf, D., Klamt, S., Centler, F., 2019. Augmenting biogas process modeling by resolving intracellular metabolic activity. Front. Microbiol. 10. https://doi.org/10.3389/fmicb.2019.01095

Westerholm, M., Dolfing, J., Schnürer, A., 2019. Growth Characteristics and Thermodynamics of Syntrophic Acetate Oxidizers. Environ. Sci. Technol. 53, 5512-5520. https://doi.org/10.1021/acs.est.9b00288

Yentekakis, I. V., Goula, G., 2017. Biogas management: Advanced utilization for production of renewable energy and added-value chemicals. Front. Environ. Sci. https://doi.org/10.3389/fenvs.2017.00007

Zacher, B., Lidschreiber, M., Cramer, P., Gagneur, J., Tresch, A., 2014. Annotation of genomics data using bidirectional hidden Markov models unveils variations in Pol 

II transcription cycle. Mol. Syst. Biol. 10, 768. https://doi.org/10.15252/msb.20145654

1052 Zhu, X., Campanaro, S., Treu, L., Kougias, P.G., Angelidaki, I., 2019. Novel ecological 1053 insights and functional roles during anaerobic digestion of saccharides unveiled 1054 by genome-centric metagenomics. Water Res. 151, 271-279. 1055 https://doi.org/10.1016/j.watres.2018.12.041

1056 Zhu, X., Campanaro, S., Treu, L., Seshadri, R., Ivanova, N., Kougias, P.G., Kyrpides, 1057 N., Angelidaki, I., 2020. Metabolic dependencies govern microbial syntrophies 1058 during methanogenesis in an anaerobic digestion ecosystem. Microbiome 8. 1059 https://doi.org/10.1186/s40168-019-0780-9

1060 Zomorrodi, A.R., Maranas, C.D., 2012. OptCom: A multi-level optimization framework 1061 for the metabolic modeling and analysis of microbial communities. PLoS Comput. 1062 Biol. 8. https://doi.org/10.1371/journal.pcbi.1002363

1063 